\title{
Cortical Stimulation Evokes Abnormal Responses in the Dopamine-Depleted Rat Basal Ganglia
}

\author{
Hitoshi Kita and Takako Kita \\ Department of Anatomy and Neurobiology, College of Medicine, The University of Tennessee Health Science Center, Memphis, Tennessee 38163
}

\begin{abstract}
The motor cortex (MC) sends massive projections to the basal ganglia. Motor disabilities in patients and animal models of Parkinson's disease (PD) may be caused by dopamine (DA)-depleted basal ganglia that abnormally process the information originating from MC. To study how DA depletion alters signal transfer in the basal ganglia, MC stimulation-induced (MC-induced) unitary responses were recorded from the basal ganglia of control and 6-hydroxydopamine-treated hemi-parkinsonian rats anesthetized with isoflurane. This report describes new findings about how DA depletion alters MC-induced responses. MC stimulation evokes an excitation in normally quiescent striatal (Str) neurons projecting to the globus pallidus external segment (GPe). After DA-depletion, the spontaneous firing of Str-GPe neurons increases, and MC stimulation evokes a shorter latency excitation followed by a long-lasting inhibition that was invisible under normal conditions. The increased firing activity and the newly exposed long inhibition generate tonic inhibition and a disfacilitation in GPe. The disfacilitation in GPe is then amplified in basal ganglia circuitry and generates a powerful long inhibition in the basal ganglia output nucleus, the globus pallidus internal segment. Intra-Str injections of a behaviorally effective dose of DA precursor L-3,4-dihydroxyphenylalanine effectively reversed these changes. These newly observed mechanisms also support the generation of pauses and burst activity commonly observed in the basal ganglia of parkinsonian subjects. These results suggest that the generation of abnormal response sequences in the basal ganglia contributes to the development of motor disabilities in PD and that intra-Str DA supplements effectively suppress abnormal signal transfer.
\end{abstract}

\section{Introduction}

Motor disabilities in patients and animal models of Parkinson's disease (PD) may be due to abnormal and/or diminished capability of data processing in dopamine (DA)-depleted basal ganglia. Data processing may be compromised by alteration of synaptic responses, generation of abnormal burst activity, and synchronized oscillations in the basal ganglia (Filion, 1979; Wichmann et al., 1994; Bevan et al., 2002, 2006; Boraud et al., 2002; Dejean et al., 2008; Israel and Bergman, 2008; Gatev and Wichmann, 2009). Because many in vitro results showed altered synaptic responses in striatum (Str) after DA depletion or application of DA-receptor (DA-R) agonists and antagonists (Flores-Hernández et al., 1997; Spencer and Murphy, 2000; Bamford et al., 2004; Flores-Barrera et al., 2010; Surmeier et al., 2010), it seems possible that altered Str synaptic responses trigger abnormal data processing in the basal ganglia.

The Str is an input nucleus of the basal ganglia and receives glutamatergic projections from the entire cortex and some thalamic nuclei. DA depletion has been shown to increase responsiveness to excitatory inputs of one type of Str projection neurons

\footnotetext{
Received Feb. 20, 2011; revised June 2, 2011; accepted June 4, 2011.

Author contributions: H.K. designed research; H.K. and T.K. performed research; H.K. and T.K. analyzed data; H.K. wrote the paper.

This work was supported by the National Institute of Neurological Disorders and Stroke Grants NS47085 and NS-57236. We thank R. Kita for editing the manuscript and J. Butler for technical assistance.

Correspondence should be addressed to Hitoshi Kita, Department of Anatomy and Neurobiology, College of Medicine, The University of Tennessee Health Science Center, 855 Monroe Avenue, Memphis, TN 38163. E-mail: hkita@uthsc.edu.

DOI:10.1523/JNEUROSCI.0915-11.2011

Copyright $\odot 2011$ the authors $\quad 0270-6474 / 11 / 3110311-12 \$ 15.00 / 0$
}

that project only to globus pallidus external segment (GPe; also called globus pallidus in rodents) while decreasing that of another projecting to GPe, globus pallidus internal segment (GPi; entopeduncular nucleus), and the substantia nigra pars reticulata $(\mathrm{SNr})$ by changing their synaptic activity and membrane properties (Hernandez-Lopez et al., 2000; Pang et al., 2001; West and Grace, 2002; Mallet et al., 2006; Shen et al., 2008; Flores-Barrera et al., 2010; Surmeier et al., 2010). It is possible that these changes in Str simply increase disynaptic motor cortex (MC)-Str-GPe inhibition and decrease MC-Str-GPi inhibition (Nambu et al., 2005). However, involvement of other possible factors should be also considered. For instance, MC stimulation evokes EPSPs followed by a long hyperpolarization in Str projection neurons (Wilson et al., 1983), but the hyperpolarization is invisible to its postsynaptic neurons because the majority of the Str neurons are quiescent. In contrast, the long inhibition evoked in DA-depleted Str neurons with increased background firing will be visible to its postsynaptic neurons as a disinhibition. To examine this possibility, MCinduced responses of basal ganglia neurons in control and 6-hydroxdopamine (6-OHDA)-treated rats (6-OHDA rats), a rodent model of $\mathrm{PD}$, were recorded in vivo.

This is the first documented report that increased spontaneous firing of Str-GPe neurons contributes substantially to the alteration of MC-induced responses in both segments of globus pallidus and subthalamic nucleus (STN) after DA depletion. To study these results further, we analyzed effects of intra-Str injection of a behaviorally effective dose of DA precursor L-3,4dihydroxyphenylalanine (L-DOPA) on the altered MC-induced responses in GPe and GPi. The local application of L-DOPA was 
used to selectively activate DA-Rs in Str but not in other brain areas. This report provides evidence that intra-Str L-DOPA can reverse changes in $\mathrm{MC}$-induced response sequences in basal ganglia that are attributed to the increased spontaneous firing of Str-GPe neurons. This suggests that generation of abnormal response sequences in the basal ganglia may be a contributor to motor disabilities in PD and that treatment to reduce tonic Str-GPe inhibition may help to restore motor function.

\section{Materials and Methods}

The experiments were performed in adult male Sprague Dawley rats (300-380 g) in compliance with the National Institutes of Health Guide for the Care and Use of Laboratory Animals and the University of Tennessee Health Science Center Guide for the Use and Care of Laboratory Animals in Research.

Behavioral tests. All rats received two kinds of behavioral tests two times before and two to four times after the DA depletion surgery described below. The rotational asymmetry test monitored ambulatory movements of rats in a 1-m-diameter chamber for $15 \mathrm{~min}$ using a video monitor system (Viewer; Biobserve), which could identify and track the rats' head, body, and tail positions frame by frame (30 frames/s). The body location data were used to calculate the distance and direction of the body movement between frames. For the limb use asymmetry test, rats were placed in a $28-\mathrm{cm}-$ diameter acrylic cylinder and videotaped for 10 min. The number of times the left and right forelimbs touched the wall of the cylinder were counted.

DA depletion and head-holder installation. After the behavioral tests, 10 rats were anesthetized with a mixture of ketamine $(85 \mathrm{mg} / \mathrm{kg}$, i.p. $)$ and xylazine $(15 \mathrm{mg} / \mathrm{kg}$, i.p.), treated with desmethylimipramine $(25 \mathrm{mg} / \mathrm{kg}$, i.p.) and pargyline ( $5 \mathrm{mg} / \mathrm{kg}$, i.p.), and placed in a stereotaxic apparatus. A craniotomy was performed on the skull, and 6-OHDA ( $8 \mu \mathrm{g}$ in $4 \mu \mathrm{l}$ of saline containing $0.1 \%$ ascorbic acid) in six rats or the vehicle in four rats was injected into the left ascending mesotelencephalic DA-bundle. The 6-OHDA or vehicle was delivered slowly over a period of $10 \mathrm{~min}$ through a glass micropipette (tip diameter, $\sim 50 \mu \mathrm{m}$ ) glued to the needle of a $10 \mu \mathrm{l}$ Hamilton syringe. The syringe plunger was advanced by a pulse motordriven actuator. After 2 weeks of recovery, the behavioral tests were performed again. Four of the six 6-OHDA rats developed a strong left turn preference in the rotational asymmetry test and a strong left forelimb use preference $(<0.2$ right/left touches in the cylinder test). The 6-OHDA rats that failed to meet these behavioral criteria were rejected for unit recording study. None of the four rats injected with the vehicle developed behavioral asymmetries.

After the behavioral tests, a second surgery was performed on the control and 6-OHDA rats. The rats were anesthetized with a mixture of ketamine $(85 \mathrm{mg} / \mathrm{kg}$, i.p. $)$ and xylazine $(15 \mathrm{mg} / \mathrm{kg}$, i.p. $)$ and mounted on a stereotaxic apparatus. To fix the rats' heads painlessly to a stereotaxic frame, a plastic head holder made with polycarbonate and stainless steel pipes was placed on the dorsal surface of the skull and secured with dental acrylic. Also, a craniotomy was performed over the lateral agranular cortex, which is the MC of rodents, and a parallel bipolar stimulus electrode $(100 \mu \mathrm{m}$ insulated stainless steel wires with tip separation of $0.6-$ $0.7 \mathrm{~mm}$ ) was implanted. For three rats, a pair of silver ball electrodes was also placed on the dura matter, one covering the right frontal agranular cortex and the other covering the cerebellum to monitor the electrocorticogram. Another craniotomy was performed over the pallidum and
STN for recording electrode penetration (Fig. $1 A$ ). A thin layer of dental acrylic was placed over the recording hole until needed for the recording sessions. After the surgery, the rats were monitored until recovery from anesthesia. If rats showed any sign of pain, buprenorphine (0.01-0.05 $\mathrm{mg} / \mathrm{kg}$, i.p.) was administered.

Unit recordings. Unit recordings began 5-7 d after installation of the head holder and stimulus electrodes. For the recordings, the rats were anesthetized with $2.5 \%$ isoflurane in oxygen and mounted on the stereotaxic device using the head holder. The use of isoflurane allowed for quick adjustment of the level of anesthesia. During the recording, the rats were maintained with $0.5-1.0 \%$ isoflurane that was adjusted to eliminate spontaneous whisker movements as well as any body movement to MC stimulation. Under these conditions, the rats did not show movement responses to light touch but responded to painful stimuli such as a foot pinch. The level of anesthesia judged by electrocorticogram observation was stage 2 or 3 sleep but not slow-wave sleep (data not shown). These levels were chosen to avoid the induction of strong, synchronized, slow oscillations during the slow-wave sleep that is induced by higher isoflurane doses. On the opposite end of the spectrum, these levels also eliminated potential activity changes of the cortex and basal ganglia induced by sensory afferent inputs activated by spontaneous or MC stimulationinduced (MC-induced) movement of body parts with lower isoflurane doses.

Single-unit activities of Str, STN, and pallidal neurons were recorded with insulated tungsten or Elgiloy alloy microelectrodes with AC resistance of 1-2.5 M $\Omega$. For juxtacellular labeling of Str neurons, glass micropipettes filled with $1 \mathrm{M} \mathrm{NaCl}$ and $2 \%$ Neurobiotin were used, and recorded neurons were labeled by a method described previously (Pinault, 1996). The spontaneous unit activity and responses to MC stimulation were amplified, passed through a $0.7-2 \mathrm{kHz}$ bandpass filter 
(Model 1800, home-modified; A-M Systems), and converted into digital data by a homemade window discriminator. The parameters of MC stimulation were $300 \mu$ s duration single pulse, strength $80 \mu \mathrm{A}$, and interstimulus interval of $1.7 \mathrm{~s}$. These parameters were adapted from previous studies (Wilson et al., 1883; Kita, 1992; Fujimoto and Kita, 1993; West and Grace, 2002). Digitized units, trigger signals, and electrocorticograms were recorded using a computer. The pallidal and STN neurons were identified by their firing patterns (Fujimoto and Kita, 1993) and by $\mathrm{x}$-ray images of the skull and the electrode (Fig. $1 A$ ). The locations were also identified histologically at the end of the experiments as described below.

Analysis of unit activity. STN and pallidal neurons were recorded from every rat, and Str neurons were recorded from two control and two 6-OHDA rats. The data obtained from the rats were pooled into control and 6-OHDA groups because the firing property and MC-induced response patterns obtained from anesthetized rats within each group were similar. The existence of the refractory period in single-unit recordings was tested by calculating an autocorrelogram online for $50 \mathrm{~s}$ of digitized spikes for pallidal and STN neurons and for $120 \mathrm{~s}$ of digitized spikes for Str neurons. The digitized recordings were also used calculate the rate and the burst index of firing of neurons. Bursts were detected using the "surprise" method of Legéndy and Salcman (1985), with the surprise value $\geq 3$ and the number of spikes $\geq 3$ in a burst. These values were adopted from an extensive study of burst activity in monkey pallidum and STN (Wichmann and Soares, 2006). The proportion of spikes in bursts (\%) was chosen as the index because this method has been frequently used in other studies.

MC-induced neuronal responses were assessed by constructing poststimulus time histograms (PSTHs) with $1 \mathrm{~ms}$ bin widths (unless noted otherwise) and 100 stimulation trials. The mean firing rate during the $100 \mathrm{~ms}$ preceding the stimulation was considered to be the baseline. For the recording from excitatory responses from silent or low spontaneously active Str neurons, single-unit recording was verified by the existence of a refractory period in the autocorrelogram calculated from digitized spikes during 100 stimulation trials. The components of MC-induced responses in the pallidum and STN include an early excitation (also called first excitation), a short inhibition (first inhibition), a late excitation (second excitation), a long inhibition (second inhibition), and a slow excitation (Fujimoto and Kita, 1993; Maurice et al., 1998; Ryan and Clark, 1991, 1992) (Fig. 1C). Response components observed in Str include a short duration excitation, a long inhibition, and a rebound-like excitation. The criterion for definition of the early and the late excitations was that the firing rate of three consecutive bins of the PSTH exceeded the $95 \%$ confidence interval of the baseline (one-tailed $t$ test for each bin). The criterion for the short inhibition was same with the excitation criterion for the neurons having a prestimulus firing rate higher than the $95 \%$ confidence interval. For the neurons having a lower firing rate, the short inhibition criterion was at least six consecutive bins of zero firing, which was based on the following two reasons: (1) most of the short inhibitions detected in both pallidum and STN by the $95 \%$ confidence interval method had more than six bins and (2) there was no occurrence of consecutive zero six bins at prestimulus portion of PSTHs. In many neurons, the trough between the early and late excitations did not meet the criteria for the inhibition. In these neurons, the lowest point of the trough was defined as the separation point between the early and late excitations. The criteria for the long inhibition and the slow excitation were that the moving average with a $10 \mathrm{~ms}$ window exceeded $50 \%$ of the average prestimulus rate and that the response duration exceeded $30 \mathrm{~ms}$ (i.e., $3 \times$ the averaging window). These criteria also defined the onset and offset times of the long inhibition, which are required for comparisons of response parameters. The reason for the 50\% threshold was that the onset and the offset of the long inhibition with $>50 \%$ (i.e., relatively strong response) rate changes can be relatively easily defined. Test comparisons of prestimulus and defined slow response parts of PSTH in several STN neurons by Mann-Whitney $U$ test resulted in significant differences with $p<0.001$ (data not shown). The strength of the response was a sum of the firings in the response that were above or below the prestimulus mean rate (i.e., the area of the response) (Fig. 1C). The existence of identifiable response components for every recorded neuron was examined, and if a neuron had at least one response component that met the criteria, the neuron was labeled MC-responsive.

Intrastriatal injections. The intra-Str injection of L-DOPA methyl ester hydrochloride (methyl-L-DOPA, $4 \mu \mathrm{g}$ in $0.2 \mu \mathrm{l}$ of saline) or muscimol $(0.04 \mu \mathrm{g}$ in $0.2 \mu \mathrm{l}$ of saline) was performed in a similar manner to the 6-OHDA injection described above. The injection site in Str was first determined by observing $\mathrm{MC}$-induced unit responses recorded through the injection micropipette and then the drugs were injected slowly for a total of $0.2 \mu \mathrm{l}$ over $5 \mathrm{~min}(0.04 \mu \mathrm{l} / \mathrm{min})$ with an electric actuator on the microsyringe plunger. A slow injection rate was used to control spreading of drug, in particular, to avoid spreading to the GPe. The drug injection was performed with at least a $3 \mathrm{~d}$ interval between injections. The methyl-L-DOPA dose was determined by the ambulatory direction and distance tests on two 6-OHDA rats, as described in Results, below. The muscimol dose was based on previous observations that $0.04 \mu \mathrm{g}$ of muscimol blocked MC-induced excitation in an $\sim 0.5 \mathrm{~mm}$ radius of the injection area (data not shown). The present data confirmed that muscimol did not spread to GPe and suppress the firing activity. The absence of spreading into GPe also suggests a possibility that muscimol and methyl-L-DOPA did not cover the entire Str and may have produced submaximal effects in GPe. The spontaneous firing rate, the amplitudes, and the duration of MC-induced responses obtained immediately before and 10-15 min after completion of drug injection, at which time the effects of local drug injections on neural activity and animal behavior are stable and near maximum (Nambu et al., 2000; Kita et al., 2004), were evaluated by paired $t$ test, and the data are expressed as mean \pm SD.

Histology. For the final experiment, two to three recording sites were marked by passing a cathodal current $(10 \mu \mathrm{A}$ for $5 \mathrm{~s})$ through metal recording electrodes. Approximately 1-2 d after the lesion, rats were deeply anesthetized with a mixture of ketamine $(100 \mathrm{mg} / \mathrm{kg})$ and xylazine $(20 \mathrm{mg} / \mathrm{kg}$ ) and were perfused through the heart with $10-20 \mathrm{ml}$ of isotonic saline followed by a fixative. The fixative was a mixture of $4 \%$ formaldehyde and $0.2 \%$ picric acid in a $0.12 \mathrm{M}$ sodium phosphate buffer (200-300 ml, pH 7.4). After perfusion, the brains were removed and postfixed overnight at $4^{\circ} \mathrm{C}$ and then equilibrated in $10 \%$, followed by $30 \%$, phosphate buffered, $\mathrm{pH} 7.4$, sucrose solution. The brains were cut into $40 \mu \mathrm{m}$ sagittal sections on a freezing microtome. Sections from neurobiotin-injected brains were incubated for $5 \mathrm{~h}$ in PBS containing $0.5 \%$ skim milk, $0.05 \%$ Triton X-100, and ABC Elite (1:150 dilution; Vector). After several washes with PBS, the sections were incubated for 10-20 min in 0.05 M Tris- $\mathrm{HCl}$ buffered saline, $\mathrm{pH} 7.6$, containing $0.04 \%$ diaminobenzidine tetrahydrochloride, $0.04 \% \mathrm{NiCl}_{2}$, and $0.001 \% \mathrm{H}_{2} \mathrm{O}_{2}$ to reveal biotin containing neurons. Some of the sections were immunostained for tyrosine hydroxylase (TH), and others were stained with $1 \%$ cresyl violet to verify the recording sites (Fig. $1 B$ ). The sections for $\mathrm{TH}$ staining were incubated first for $5 \mathrm{~h}$ in PBS containing $0.5 \%$ skim milk and $0.05 \%$ Triton X-100 and then for $48 \mathrm{~h}$ in PBS containing a monoclonal mouse anti-TH antibody (1:4000; Sigma), 0.5\% skim milk, and $0.05 \%$ Triton X-100. Subsequently, the sections were incubated with biotinylated goat anti-mouse IgG antibody (1:200 dilution; Vector) in the PBS incubation medium for $2 \mathrm{~h}$. The sections were treated with $\mathrm{ABC}$ and diaminobenzidine tetrahydrochloride as described for the neurobiotin sections. The sections were mounted on gelatin-coated slides, airdried, dehydrated in graded alcohols to xylene, and coverslipped. All the chemicals used for making the fixative, buffers, and other histological solutions were obtained from Sigma-Aldrich.

\section{Results}

\section{MC-induced responses of GPe neurons}

Previous studies showed that DA depletion increases the responsiveness of Str-GPe neurons to excitatory inputs (HernandezLopez et al., 2000; Pang et al., 2001; West and Grace, 2002; Mallet et al., 2006; Shen et al., 2008; Flores-Barrera et al., 2010; Surmeier et al., 2010). The aim of the present GPe recordings was to examine how this increased responsiveness affects MC-induced responses in GPe. Previous studies have also shown that MC stimulation typically induces a sequence of responses consisting of an early excitation, a short inhibition, and a late excitation, of 
Table 1. Response parameters of MC-responsive GPe neurons

\begin{tabular}{|c|c|c|c|}
\hline & Control $(N=39)$ & & $6-\mathrm{OHDA}(N=42)$ \\
\hline Average firing rate & $30.4 \pm 11.4$ & ns & $27.3 \pm 9.8$ \\
\hline Burst index, $\%$ & $12.6 \pm 14.9$ & $p<0.0001$ & $45.6 \pm 16.2$ \\
\hline Early excitation, $n / N(\%)$ & $31 / 39(79.5 \%)$ & & $26 / 42(61.9 \%)$ \\
\hline Latency (ms) & $7.4 \pm 2.2$ & ns & $6.8 \pm 1.5$ \\
\hline Duration (ms) & $4.9 \pm 2.1$ & ns & $4.2 \pm 1.1$ \\
\hline Strength (spikes) & $36.2 \pm 25.9$ & ns & $30.3 \pm 20.1$ \\
\hline Short inhibition & $21 / 39(53.9 \%)$ & & $32 / 42(76.2 \%)$ \\
\hline Latency & $13.4 \pm 1.5$ & $p<0.02$ & $12.0 \pm 1.9$ \\
\hline Duration & $11.2 \pm 3.7$ & ns & $10.6 \pm 4.3$ \\
\hline Strength & $28.9 \pm 14.6$ & ns & $26.8 \pm 12.6$ \\
\hline Late excitation & $32 / 39(82.1 \%)$ & & $41 / 42(97.6 \%)$ \\
\hline Latency & $27.0 \pm 4.9$ & ns & $26.4 \pm 7.5$ \\
\hline Duration & $39.8 \pm 51.6$ & $p<0.0001$ & $129.0 \pm 82.6$ \\
\hline Strength & $180.3 \pm 303.1$ & $p<0.0001$ & $412.5 \pm 301.6$ \\
\hline Long inhibition & 4/39 (10.3\%) & & $5 / 42(11.9 \%)$ \\
\hline Latency & $45.8 \pm 11.7$ & ns & $33.6 \pm 7.5$ \\
\hline Duration & $145.3 \pm 59.0$ & ns & $116.4 \pm 30.8$ \\
\hline Strength & $215.1 \pm 174.7$ & ns & $252.8 \pm 111.3$ \\
\hline
\end{tabular}

Statistics: Mann-Whitney U test. $N$, Number of neurons; ns, not significant.

which the short inhibition was mediated by Str while both the early and late excitations were mediated by STN (Ryan and Clark, 1991; Kita, 1992; Yoshida et al., 1993; Nambu et al., 2000; Kita et al., 2004). These results were incorporated to develop the hypothesis that $\mathrm{MC}$-induced short inhibition increases after DA depletion.

The GPe neurons included in this report were of the highfrequency firing with pause type, which are the most common type found in GPe (DeLong, 1971). The neurons with a slow, $<10$ $\mathrm{Hz}$, rhythmic firing of large, broad spikes that were encountered mainly in the caudomedial and ventral regions of the nucleus were excluded from the present analysis. A total of 39 and $42 \mathrm{MC}$ responsive GPe neurons in control and in 6-OHDA rats, respectively, were recorded. The mean firing rate was unchanged, though the burst index significantly increased in 6-OHDA rats (Table 1). These average firing rates were only slightly lower than in paralyzed rats (Kelland et al., 1995), but much lower than in awake primates (Albin et al., 1989; Bergman et al., 1994; Ni et al., 2000; Kita et al., 2004; Wichmann and DeLong, 2006), probably due to the isoflurane anesthesia given to the rats.

Figures 2, $A$ and $B$, shows the two major response patterns observed in GPe of control rats. Thirteen of 39 neurons responded with an early excitation, a short inhibition, and a late excitation (Fig. $2 \mathrm{~A}$ ), and nine responded with an early excitation followed by a late excitation (Fig. $2 B$ ). In the latter neurons, the trough between the early and the late excitations did not meet the criteria for an inhibition, even though an inhibition evoked by Str inputs may have contributed to form the trough. The remaining 17 neurons exhibited other combinations of response components (data not shown). Of the 42 MC-responsive GPe neurons recorded in 6-OHDA rats, 20 responded with an early excitation, a short inhibition, and a late excitation (Fig. 2C), and 12 responded with a short inhibition followed by a late excitation (Fig. $2 D)$. The remaining 10 neurons exhibited other combinations of the response components.

Table 1 compares the parameters of the MC-induced response components. The population of neurons showing a short inhibition slightly increased from 21 of 39 (53.9\%) neurons in the control group to 32 of $42(76.2 \%)$ in the 6-OHDA group. Also, the latency of the inhibition decreased in the 6-OHDA rats (Table 1), while their strength was unchanged. These observations do not provide strong support for the hypothesis that the strength of the inhibition increases after DA depletion. Instead, the most notable difference was the significant increase in the duration and strength of the late excitation in 6-OHDA rats (Table 1). The population responses of all GPe neurons obtained from control and 6-OHDA rats also show a shortening of the latency of the short inhibition and a clear increase of the late excitation (Fig. $2 E$ ) in the 6-OHDA rats. The recordings in STN and GPi described below suggest that the increased late excitation significantly alters MC-induced responses in these nuclei. As a result, experiments to identify the mechanism driving this increased late excitation were performed.

\section{Effects of NMDA receptor blockage in GPe}

A possible mechanism for the strong late excitation in GPe may be an augmentation of the response mediated by NMDA-R. To test this possibility, the NMDA-R antagonist 3-(2-carboxypiperazin-4yl) propyl-1-phosphonic acid (CPP; $50 \mathrm{ng}$ in $0.2 \mu \mathrm{l}$ of saline, 0.04 $\mu \mathrm{l} / \mathrm{min}$ ) was injected into GPe of 6-OHDA rats. After the removal of the injection pipette, neurons responding with an early excitation, a short inhibition, and a late excitation were recorded from the area within a $0.5 \mathrm{~mm}$ radius of the injection site within $30 \mathrm{~min}$ of the injection. The mean firing rate of these eight neurons was $17.7 \pm 4.4 \mathrm{~Hz}$, which was lower than that obtained under control conditions, as expected from the results of a previous study (Kita et al., 2004). Figure $2 \mathrm{~F}$ compares the population PSTH obtained after CPP injection with respect to the PSTH shown in Figure 2C, in which the amplitude was reduced to make the average prestimulus firing rates of both PSTHs equal. The long duration of the late excitation was still observed after CPP, although the rising phase of the excitation was much slower than those recorded without CPP. These observations suggest that NMDA-R activation contributes only to the very early phase and not to the late phase of the late excitation that developed after DA depletion.

\section{MC-induced responses of STN neurons}

The second possibility was that MC-induced excitation in STN increased after DA depletion and that the increased excitation drove the strong late excitation in GPe. This possibility was based on the previous studies showing that the major driving forces for both the early and late excitations in GPe originated from STN (Nambu et al., 2000; Kita et al., 2004; Kita, 1992, 1994), that STN neurons in PD patients respond strongly to sensory stimulation (Rodriguez-Oroz et al., 2001), and that the responsiveness of the cortico-STN-SNr pathway increased after DA depletion (Dejean et al., 2008). In vitro studies also have shown that DA agonists altered the membrane properties and the responsiveness of STN neurons to glutamate and GABA (Gajendiran et al., 2005; Shen and Johnson, 2005).

To test this possibility, 46 and 44 MC-responsive STN neurons in control and 6-OHDA rats, respectively, were observed. A majority of the neurons (27 of 46) recorded in control rats evoked an early excitation, a late excitation, and a long inhibition after MC stimulation (Fig. 3A). In these neurons, the trough between the early and the late excitations did not meet the criteria for an inhibition. Five neurons evoked an early excitation, a short inhibition, and a late excitation (Fig. 3B). These response patterns were very similar to previous reports (Kitai and Deniau, 1981; Ryan and Clark, 1992; Fujimoto and Kita, 1993; Maurice et al., 1998). The remaining 14 neurons exhibited other combinations of the response components. The STN neurons in 6-OHDA rats had a significantly higher average firing rate and a higher average burst index than those in the control rats (Table 2). Like the 


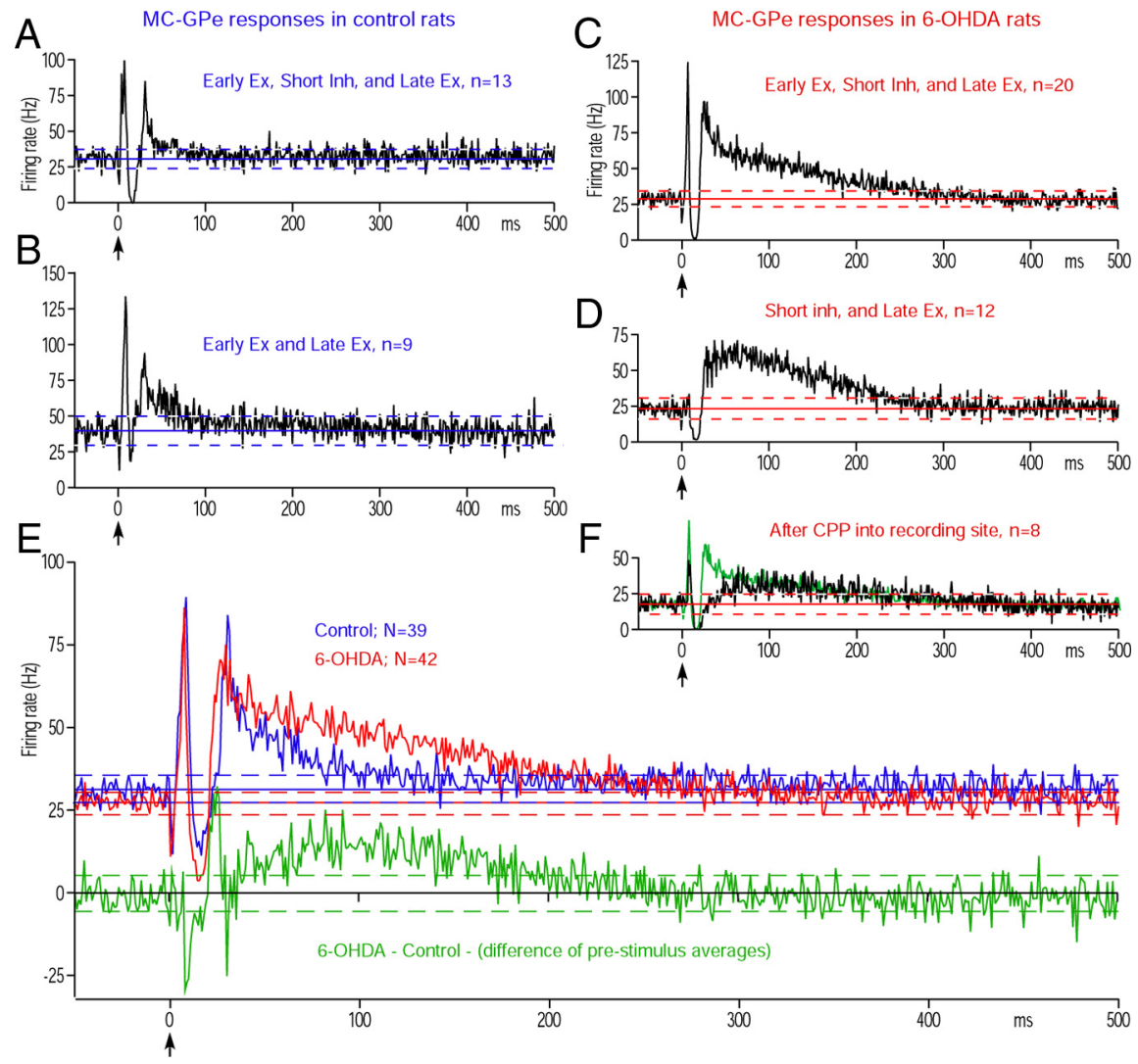

Figure 2. MC-induced responses in GPe. $\boldsymbol{A}, \boldsymbol{B}$, Population PSTHs of two major response patterns obtained in control rats. In these and subsequent figures, population PSTH (i.e., average PSTH of multiple neurons) was constructed from 100 stimulation trials. The bin width of PSTHs is $1 \mathrm{~ms}$ unless otherwise noted, and $\mathrm{MC}$ stimulation was given at time $=0$ (arrow). The response pattern, the number of neurons included in each population PSTH, and the average prestimulus firing frequency with $95 \%$ confidence interval (solid line and dashed lines) are shown in each figure. Ex., Excitation; Inh., inhibition. C, D, Population responses of two major response patterns obtained in 6-OHDA rats. Ex., Excitation; Inh., inhibition. E, Population PSTHs of all MC-responsive GPe neurons in control and 6-OHDA rats. The green PSTH shows the delta between the control and 6-OHDA PSTHs. $\boldsymbol{F}$, A population PSTH of neurons that responded with an early excitation, a short inhibition, and a late excitation in 6-0HDA rats after injection of CPP, an NMDA antagonist, into the recording sites. For comparison, the same PSTH (in green) shown in $\boldsymbol{C}$ is scaled to have the same prestimulus firing rate and overlaid.

controls, a majority of MC responsive neurons (28 of 44) in 6-OHDA rats evoked an early and a late excitation and a long inhibition (Fig. 3C). Figure 3, D and E, shows that the second and third most frequent response patterns were an early excitation and a prominent long inhibition (Fig. 3D) and a long inhibition only (Fig. $3 E$ ); this last response pattern was never observed in control rats. Table 2 and all cell population PSTHs (Fig. 3F) show that the strength of the early and the late excitations decreased in 6-OHDA rats, though the numbers of neurons evoking the early and late excitations were similar in both the control and 6-OHDA groups. These data does not support the hypothesis that the increased late excitation in the GPe was driven by an increase in the excitatory responses in STN. The most notable change was the increase in the strength of the long inhibition with an increased percentage of neurons showing the inhibition with decreased latency, increased duration, and increased strength in 6-OHDA rats (Fig. 3F; Table 2).

\section{MC-induced responses of Str neurons}

It was shown in an in vivo intracellular recording study that MCinduced excitation in Str neurons is followed by a long inhibition consisting of a GABAergic inhibition and cortical disfacilitation (Wilson et al., 1983). The long inhibition evoked in normally quiescent Str neurons is invisible to its postsynaptic neurons. If DA depletion increases the spontaneous firing of Str neurons projecting to $\mathrm{GPe}$, as suggested by previous studies (Hernandez-Lopez et al., 2000; Pang et al., 2001; Tseng et al., 2001; West and Grace, 2002; Mallet et al., 2006; Shen et al., 2008), the MC-induced long inhibition in the Str neurons should become visible as a disinhibition in GPe. To test this possibility, the effect of DA depletion on the spontaneous firing rate of Str neurons and whether or not MC stimulation induced a long inhibition in the spontaneously active neurons were studied.

The current results support those from previous studies showing that MC stimulation evoked a strong excitation in lowactivity neurons in the motor territory of Str in control rats. The spontaneous firing rate of the neurons recorded was $0-3.7 \mathrm{~Hz}$ (mean, $0.67 \pm 0.98 \mathrm{~Hz}$ ), and the mean latency of the excitation was $10.5 \pm 2.1 \mathrm{~ms}$ $(n=23)$ (Fig. 4A). MC-induced excitation disclosed numerous MC-responsive, normally silent neurons in succession during advancement of the recording electrode. In 6-OHDA rats, neurons with irregular spontaneous firing with a rate of $2-11 \mathrm{~Hz}$ (mean, $6.64 \pm 2.83 \mathrm{~Hz} ; n=20$ ) were more frequently encountered among normally silent neurons than in the control rats. The firing pattern of these neurons differed from the tonic firing with large single or double spikes of putative cholinergic interneurons or the high-frequency firing with sharp spikes of putative parvalbumin-containing interneurons. The irregularly firing neurons responded with a strong excitation with a mean latency of $5.1 \pm 1.0 \mathrm{~ms}$, a long inhibition with $\sim 250 \mathrm{~ms}$ duration, and a slow excitation (Fig. $4 B$ ). The mean latency of the initial excitation was significantly shorter than control $(p<0.0001)$. Juxtacellular labeling of eight MC-responsive irregularly firing neurons with neurobiotin confirmed that they were medium spiny neurons. The axons of five neurons were traced to GPe, but the other three faded out before entering GPe (data not shown). The five axons traced to GPe formed extensive axonal arbors confined in $\mathrm{GPe}$, and none of these axons exited the GPe.

The present data of increased spontaneous activity could not be compared statistically because there were too many silent neurons that could not be counted reliably, but the data confirmed the previous suggestion that DA depletion increases spontaneous activity of some Str-GPe neurons (Pang et al., 2001; Mallet et al., 2006). The present data demonstrate new aspects that MC stimulation indeed induced a shorter latency excitation and a long inhibition in the spontaneously active Str-GPe neurons.

\section{Muscimol injection into Str}

To validate the contribution of the long inhibition in Str neurons to the generation of the strong late excitation in GPe neurons, intra-Str injections of the $\mathrm{GABA}_{\mathrm{A}}$ receptor agonist muscimol was performed. In this experiment, $\mathrm{MC}$-induced multiunit excitatory 
responses were recorded using a micropipette containing muscimol to guide the pipette into the middle of the MCresponsive area of the Str. Then, GPe neurons responding with an early excitation, a short inhibition, and a late excitation to MC stimulation were recorded before and 10-20 min after muscimol injection (0.04 $\mu \mathrm{g}$ in $0.2 \mu \mathrm{l}$ of saline, $0.04 \mu \mathrm{l} / \mathrm{min})$. Because of the selection of neurons with this particular response type, the response parameters shown in Table 3 differ from those of 6-OHDA GPe in Table 1. Injection of muscimol significantly increased the prestimulus firing rate and burst index of GPe neurons (Table 3). The increase in firing indicated that Str provides tonic inhibition to GPe and that muscimol did not spread into the GPe recording site. The absence of spreading into GPe also suggests the possibility that muscimol did not cover the entire Str and may have produced submaximal effects in GPe. The existence of the tonic inhibition is a necessary condition for a disinhibition to take place. Because of the increased firing rate, the amplitude of the excitation measured from the prestimulus firing rate decreased, resulting in a significant decrease in the duration and the strength of the late excitation (Fig. 5A, Table 3 ). These effects were consistent with the expectation that decreasing the Str-GPe background inhibition by silencing Str neurons results in decreased disinhibition in GPe. Muscimol also significantly reduced the short inhibition occurring between the early and late excitations (Fig. 5A, Table 3), which was expected because muscimol should increase the input conductance of Str neurons and decrease MC-induced excitation in Str neurons (Yoon et al., 1990).

\section{Effects of L-DOPA injection into Str}

To evaluate to what degree the changes described above could be attributed to DA depletion in Str, the acute effects of intra-Str injection of the DA precursor methyl-L-DOPA on the MCinduced responses of GPe neurons in 6-OHDA rats were examined. Other than a few microdialysis studies, there has not been any reported physiological or behavioral study that used injection of methyl-L-DOPA into the Str of rats. Therefore, a behavioral test was performed first to determine the appropriate dose of methyl-L-DOPA for the recording experiments. Two rats were prepared in a similar method to the recording rats, and various doses of methyl-L-DOPA $(0.5,1,2$, and $4 \mu \mathrm{g}$ in $0.2 \mu \mathrm{l}$ of saline, injection rate $0.04 \mu \mathrm{l} / \mathrm{min}$ ) were injected into the left $\mathrm{Str}$ (i.e., ipsilateral to 6-OHDA treatment) of rats anesthetized with $1 \%$ isoflurane. The volume of the vehicle was adapted from the muscimol experiment as described above. After the injection, the rats were removed from the head holder and allowed to recover from anesthesia. The rats started to move 1-2 min after removal of isoflurane and appeared completely recovered from anesthesia after $5 \mathrm{~min}$. The directional asymmetry test was initiated as described in the Materials and Methods, above, $10 \mathrm{~min}$ after the removal of anesthesia. The tests revealed that 2 or $4 \mu \mathrm{g}$ of methyl-
Table 2. Response parameters of MC-responsive STN neurons

\begin{tabular}{lclc}
\hline & Control $(N=46)$ & & $6-0 \mathrm{HDA}(N=44)$ \\
\hline Average firing rate & $11.8 \pm 9.1$ & $p<0.0002$ & $20.3 \pm 9.3$ \\
Burst index, \% & $35.0 \pm 16.7$ & $p<0.0001$ & $54.1 \pm 15.6$ \\
Early excitation, $n / N(\%)$ & $44 / 46(95.7 \%)$ & & $39 / 44(88.6 \%)$ \\
$\quad$ Latency (ms) & $5.5 \pm 1.3$ & $\mathrm{~ns}$ & $5.9 \pm 1.6$ \\
$\quad$ Duration (ms) & $6.5 \pm 2.2$ & $p<0.001$ & $5.0 \pm 1.8$ \\
$\quad$ Strength (spikes) & $51.3 \pm 32.3$ & $p<0.02$ & $36.7 \pm 25.6$ \\
Short inhibition & $10 / 46(21.7 \%)$ & & $5 / 44(11.4 \%)$ \\
$\quad$ Latency & $10.4 \pm 2.3$ & $\mathrm{~ns}$ & $11.2 \pm 1.3$ \\
$\quad$ Duration & $9.6 \pm 4.1$ & $\mathrm{~ns}$ & $8.4 \pm 3.6$ \\
$\quad$ Strength & $14.6 \pm 9.8$ & $\mathrm{~ns}$ & $8.5 \pm 4.8$ \\
Late excitation & $35 / 46(76.1 \%)$ & & $31 / 44(70.5 \%)$ \\
$\quad$ Latency & $19.1 \pm 3.8$ & $p<0.05$ & $16.7 \pm 3.2$ \\
$\quad$ Duration & $14.5 \pm 6.8$ & $\mathrm{~ns}$ & $11.5 \pm 5.1$ \\
$\quad$ Strength & $124.5 \pm 98.9$ & $p<0.05$ & $84.4 \pm 74.3$ \\
Long inhibition & $35 / 46(76.1 \%)$ & & $43 / 44(97.7 \%)$ \\
$\quad$ Latency & $40.1 \pm 11.9$ & $p<0.001$ & $31.5 \pm 7.3$ \\
Duration & $134.9 \pm 57.2$ & $p<0.02$ & $174.2 \pm 67.6$ \\
Strength & $104.1 \pm 57.6$ & $p<0.0001$ & $298.7 \pm 168.5$ \\
\hline
\end{tabular}

Statistics: Mann-Whitney $U$ test. $N$, Number of neurons; ns, not significant.

L-DOPA changed the preferred turning direction from the left to the right and also increased the total distance moved in the test period (none statistically observed) (Fig. $5 B, C$ ). The L-DOPA effects started to decrease $\sim 20-30$ min after the injection.

In the recording sessions, GPe neurons responding with an early excitation, a short inhibition, and a late excitation to $\mathrm{MC}$ stimulation were recorded before and 10-20 min after methyl-L- 


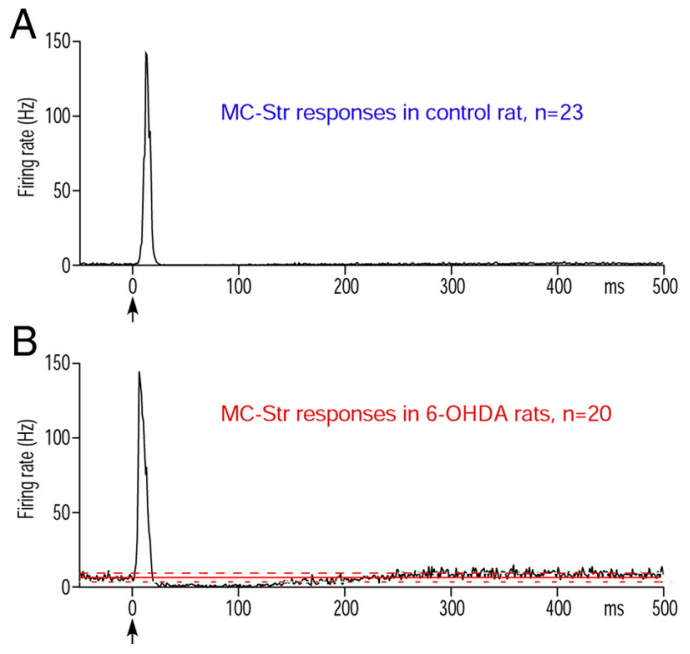

Figure 4. $M C$-induced responses in Str. $A$, A population PSTH shows MC-induced excitatory response of 23 Str neurons in control rats. $B, A$ PSTH shows a population PSTH constructed from 20 spontaneously active Str neurons recorded in 6-OHDA rats. The response consisted of an excitation, a long inhibition, and a slow excitation. The latency of these excitations was significantly shorter than in the controls.

Table 3. Effects of intra-Str injections of muscimol and methyl L-DOPA on response parameters of MC-responsive GPe neurons

\begin{tabular}{|c|c|c|c|c|c|c|}
\hline & \multicolumn{3}{|c|}{ Muscimol $(N=6)$} & \multicolumn{3}{|c|}{ Methyl-L-DOPA $(N=6)$} \\
\hline & Before & & After & Before & & After \\
\hline Average firing rate & $28.3 \pm 5.8$ & $p<0.02$ & $42.4 \pm 9.7$ & $28.7 \pm 8.6$ & $P<0.05$ & $40.6 \pm 13.6$ \\
\hline Burst index, $\%$ & $38.9 \pm 13.5$ & $p<0.001$ & $17.1 \pm 2.2$ & $43.0 \pm 8.2$ & $P<0.005$ & $25.9 \pm 11.0$ \\
\hline Early excitation, $n$ & 6 & & 6 & 6 & & 1 \\
\hline Latency (ms) & $7.3 \pm 1.0$ & ns & $8.0 \pm 1.7$ & $7.3 \pm 1.5$ & - & 8.2 \\
\hline Duration (ms) & $3.7 \pm 0.8$ & ns & $4.2 \pm 0.9$ & $3.5 \pm 0.8$ & -- & 5.2 \\
\hline Strength (spikes) & $14.6 \pm 3.7$ & ns & $21.5 \pm 11.2$ & $12.1 \pm 2.6$ & 一- & 15.2 \\
\hline Short inhibition & 6 & & 0 & 6 & & 6 \\
\hline Latency & $15.0 \pm 2.3$ & & & $14.7 \pm 1.5$ & ns & $16.2 \pm 2.0$ \\
\hline Duration & $11.0 \pm 4.6$ & & & $14.2 \pm 2.0$ & $P<0.005$ & $10.0 \pm 1.1$ \\
\hline Strength & $31.2 \pm 8.4$ & & & $40.3 \pm 13.7$ & ns & $38.1 \pm 12.8$ \\
\hline Late excitation & 6 & & 6 & 6 & & 6 \\
\hline Latency & $34.0 \pm 4.3$ & ns & $31.2 \pm 8.4$ & $34.2 \pm 5.2$ & ns & $38.7 \pm 10.8$ \\
\hline Duration & $80.0 \pm 33.6$ & $p<0.005$ & $23.8 \pm 8.9$ & $153.2 \pm 45.4$ & $P<0.02$ & $66.2 \pm 38.3$ \\
\hline Strength & $214.9 \pm 102.7$ & $p<0.02$ & $100.5 \pm 54.1$ & $457.2 \pm 189.8$ & $P<0.02$ & $222.2 \pm 116.2$ \\
\hline
\end{tabular}

Statistics: Paired $t$ test. ns, Not significant; --, not compared.

DOPA injections ( $4 \mu \mathrm{g}$ in $0.2 \mu \mathrm{l}$ of saline, $0.04 \mu \mathrm{l} / \mathrm{min}$ ). Population responses (Fig. 5D) and comparisons of response parameters (Table 3) show that methyl-L-DOPA significantly increased the prestimulus firing rate, decreased the burst index, and decreased the strength of the late excitation. The duration of the short inhibition also decreased, while the peak amplitude and strength were unchanged. These results show that in 6-OHDA rats, tonic Str-GPe inhibition, and MC-induced Str-GPe disinhibition can be removed by acute replacement of DA in Str.

\section{MC-induced responses of GPi neurons}

The MC induced responses in Str, GPe, and STN converge on GPi neurons (Fig. 7). The increased late excitation in GPe and increased long inhibition in STN should evoke a strong, long inhibition in GPi. To confirm this hypothesis, 32 and $30 \mathrm{MC}-$ responsive GPi neurons were recorded from control and 6-OHDA rats, respectively. Their mean firing rates were similar, while the burst index increased in the 6-OHDA rats (Table 4). Figure $6, A$ and $B$, shows the two most popular response patterns recorded from the control rats. MC stimulation induced combinations of an early excitation, a short inhibition, and a late exci-
A

MC-GPe responses in 6-OHDA rats
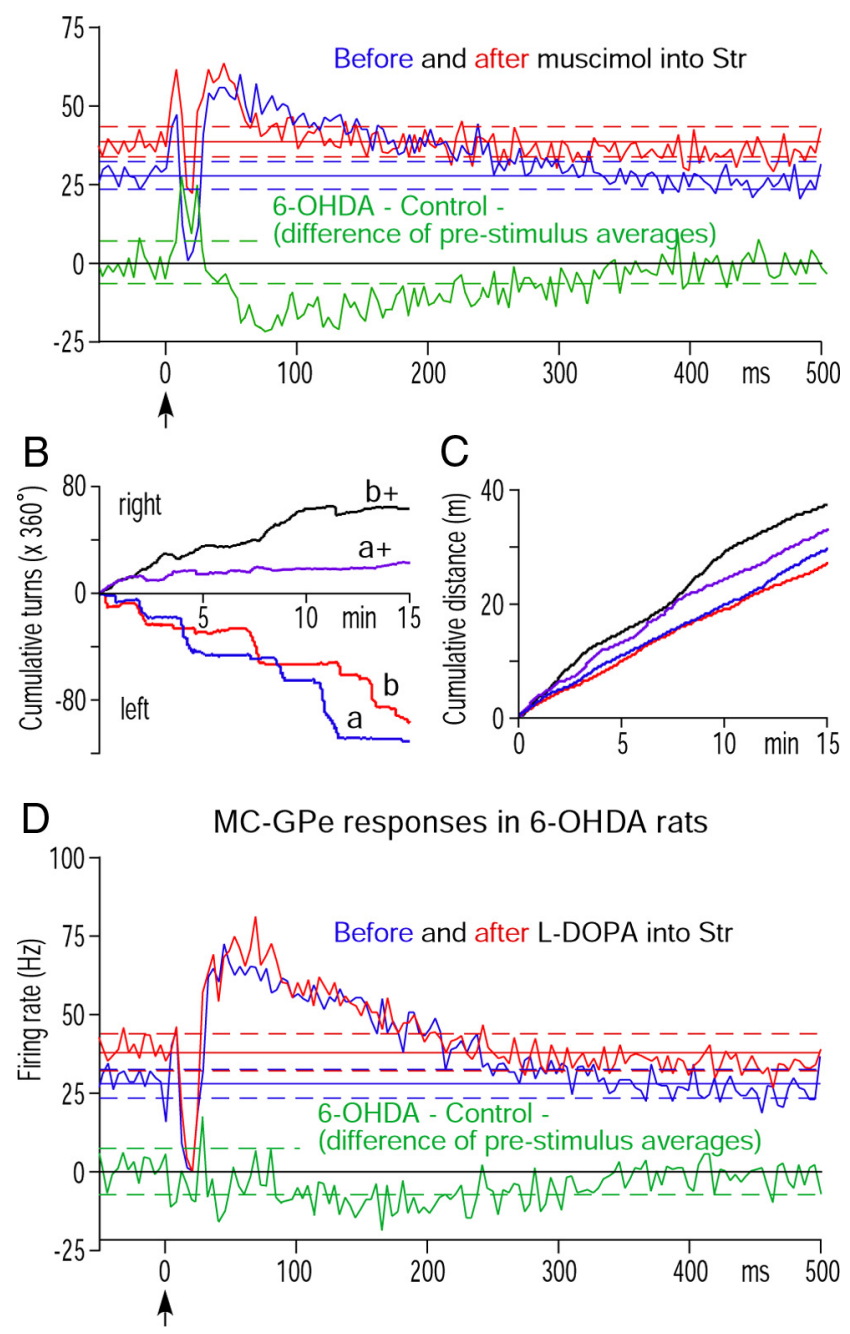

Figure 5. A, Population PSTHs compare MC-induced responses of six GPe neurons in 6-OHDA rats recorded before and after intra-Strinjection of muscimol. Neurons responding with an early excitation, a short inhibition, and a late excitation to $M C$ stimulation were selected for the experiment. $A, D$, The bin width of the PSTHs is $4 \mathrm{~ms}$ and the green PSTH shows the delta of the before and after PSTHs. B, C, Behavioral effects of intra-Str injection of methyl L-DOPA. Methyl L-DOPA altered the preferred turning direction from left to right and increased the total distance of ambulation of two 6-OHDA rats ( $a$ and $b$ are before and $a+a n d b+$ are after the injection.) D, Population PSTHs of six GPe neurons that responded with an early excitation, a short inhibition, and a late excitation to MC stimulation in 6-OHDA rats recorded before and after intra-Str injection of methyl L-DOPA.

tation in most of the neurons recorded in control rats. The response patterns observed in GPi of 6-OHDA rats differed greatly from those observed in the controls. A majority of neurons (16 of 30) evoked an early excitation followed by a strong, long inhibition (Fig. 6C). The second most popular pattern was a solo long inhibition (Fig. 6D), which was never observed in the control rats.

Table 4 compares parameters of the response components, and Figure $6 E$ compares the population PSTHs of all recorded neurons. One of the significant differences is an increased long inhibition. In control rats, a detectable long inhibition was observed in only 11 of $32(34.4 \%)$ neurons, while all $30 \mathrm{MC}$ responsive neurons in 6-OHDA rats evoked a long inhibition with significantly shorter latencies, with longer durations and greater strengths (Table 4). The shortened latency may have contributed to the significant decrease in the late excitation. Another signifi- 
cant change was a disappearance of the short inhibition. Twentytwo of $32(68.8 \%)$ neurons in the control group had the inhibition, while none were found in the 6-OHDA rats. The disappearance of the inhibition made it impossible to separate an early and a late excitation in 20 of 32 neurons. Thus, the early excitation in these neurons includes the late excitation component and also contributed to the increase in their duration and strength; because these components could not be separated, statistical comparisons were not calculated.

Intra-Str methyl-L-DOPA injection significantly altered responses of GPe neurons in 6-OHDA rats, as described above. To confirm that those changes that occurred in GPe are transmitted to GPi and also to examine whether or not an acute replacement of DA in Str can restore the short inhibition, six GPi neurons responding with an excitation and a long inhibition to MC stimulation in 6-OHDA rats were tested with intra-Str methyl-LDOPA injection using a similar method to that described above. Figure $6 F$ compares the population responses of the neurons. Methyl-L-DOPA significantly decreased the prestimulus firing rate from $28.4 \pm 2.5$ to $19.8 \pm 1.7 \mathrm{~Hz}(p<$ 0.0001 , paired $t, n=6)$, decreased the burst index from $28.4 \pm 2.5$ to $19.6 \pm$ $1.7 \%(p<0.001)$, and decreased the duration $(p<0.05)$ and the strength $(p<$ 0.01 ) of the long inhibition. These results indicate that the changes that occurred in the GPe were effectively transmitted to the GPi. In contrast, the population responses show no substantial increase in the short inhibition, though the number of neurons showing the trough separating the early and late excitations increased from two of six in the control group to all six neurons after the methyl-L-DOPA injection. There were still none that had statistically identifiable inhibition. These data, together with behavioral data, show that the behaviorally effective DA replacement in Str decreases the long duration responses and burst generation in GPi but not the short inhibition.

\section{Discussion}

Increased activity of DA-depleted Str altered response patterns of GPe, STN, and GPi neurons

This report is the first to suggest that increased spontaneous firing of Str-GPe neurons becomes a functionally dominant factor in the MC-induced responses of the basal ganglia after DA depletion. MC stimulation evokes an EPSP followed by a long hyperpolarization consisting of IPSPs and disfacilitation of cortical inputs in Str projection neurons (Wilson et al., 1983). The long hyperpolarization in normally quiescent Str neurons is invisible to its postsynaptic neurons. The intra-Str muscimol experiment demonstrated that the increased Str firing generated a background inhibition in GPe. DA depletion further enhanced the Str-GPe inhibition by removing the D2-R- and D4-R-mediated suppression (Floran et al., 1997; Cooper and Stanford, 2001; Shin et al., 2003; Watanabe et al., 2009). Because of the increased back-
Table 4. Response parameters of MC-responsive GPi neurons

\begin{tabular}{lclc}
\hline & Control $(N=32)$ & & $6-0 \mathrm{HDA}(N=30)$ \\
\hline Average firing rate & $28.0 \pm 9.1$ & Ns & $29.3 \pm 11.3$ \\
Burst index, $\%$ & $3.9 \pm 4.3$ & $p<0.0001$ & $33.4 \pm 24.9$ \\
Early excitation, $n / N(\%)$ & $20 / 32(62.5 \%)$ & & $20 / 30(66.7 \%)$ \\
$\quad$ Latency (ms) & $6.0 \pm 0.9$ & Ns & $6.9 \pm 3.3$ \\
$\quad$ Duration (ms) & $5.4 \pm 2.5$ & $p<0.01$ & $10.6 \pm 11.7$ \\
$\quad$ Strength (spikes) & $31.3 \pm 17.7$ & $p<0.05$ & $79.1 \pm 120.8$ \\
Short inhibition & $22 / 32(68.8 \%)$ & & $0 / 30(0.0 \%)$ \\
$\quad$ Latency & $13.4 \pm 1.5$ & & \\
Duration & $11.2 \pm 3.7$ & & \\
Strength & $28.9 \pm 14.6$ & & $6 / 30(20.0 \%)$ \\
Late excitation & $32 / 32(100 \%)$ & & $21.3 \pm 3.7$ \\
$\quad$ Latency & $24.3 \pm 4.2$ & Ns & $5.7 \pm 1.0$ \\
Duration & $12.4 \pm 6.1$ & $p<0.005$ & $26.5 \pm 12.3$ \\
Strength & $89.4 \pm 70.2$ & $p<0.01$ & $30 / 30(100 \%)$ \\
Long inhibition & $11 / 32(34.40 .3 \%)$ & & $31.2 \pm 10.2$ \\
Latency & $48.0 \pm 10.5$ & $p<0.0002$ & $98.6 \pm 61.8$ \\
Duration & $42.1 \pm 24.5$ & $p<0.0005$ & $230.9 \pm 179.2$ \\
Strength & $59.1 \pm 27.1$ & $p<0.0001$ & \\
\hline
\end{tabular}

Statistics: Mann-Whitney U test. ns, Not significant.
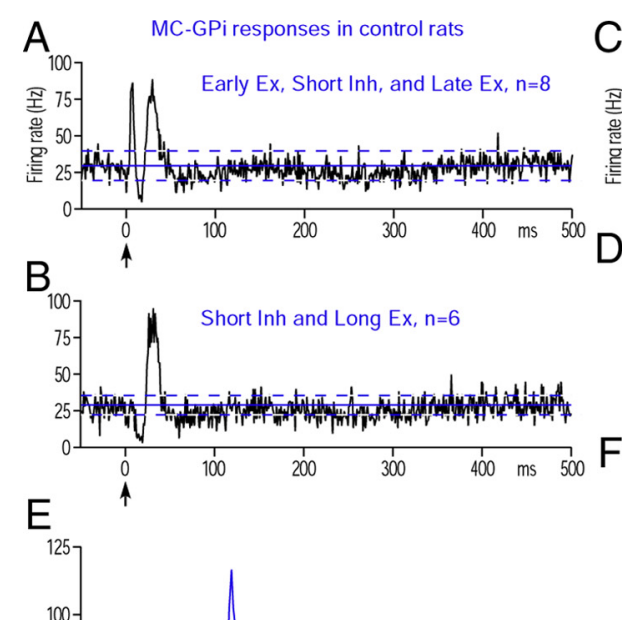

C MC-GPi responses in 6-OHDA rats
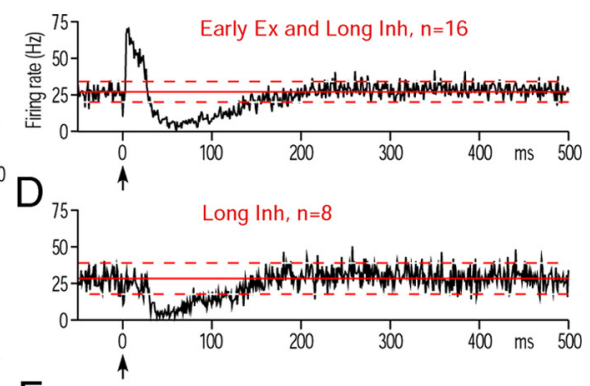

${ }^{75} 7$ Before and After L-DOPA into Str

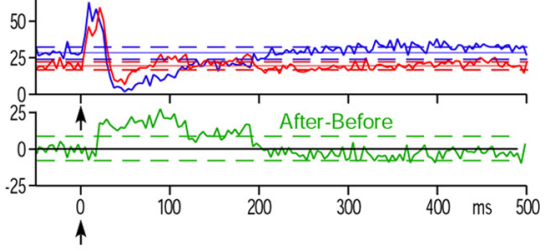

Figure 6. $M C$-induced responses in GPi. $\boldsymbol{A}, \boldsymbol{B}$, Population PSTHs of two major response patterns obtained in control rats. $\boldsymbol{C}, \boldsymbol{D}$, Population PSTHs of two major response patterns obtained in 6-OHDA rats. A majority of GPineurons recoded from 6-OHDA rats did not show the short inhibition but had a prominent long inhibition (C). The neurons showing only a long inhibition were observed in 6-OHDA rats but not in control rats (D). Ex., Excitation; Inh., inhibition. $\boldsymbol{E}$, Population PSTHs of all MC responsive GPi neurons in control and 6-OHDA rats. $\boldsymbol{E}, \boldsymbol{F}$, The green PSTH shows the delta between the control and 6-OHDA PSTHs. $\boldsymbol{F}$, Population PSTHs of six GPi neurons that responded with an early excitation and a long inhibition to MC stimulation in 6-OHDA rats recorded before and after intra-Str injection of methyl L-DOPA. The bin width of the PSTHs in $\boldsymbol{F}$ is $4 \mathrm{~ms}$.

ground inhibition, the MC-induced long hyperpolarization in Str-GPe neurons becomes visible to postsynaptic GPe neurons as a disinhibition. The disinhibition could be brought on by the Str neurons projecting solely to GPe and/or by Str-GPi/SNr neurons 


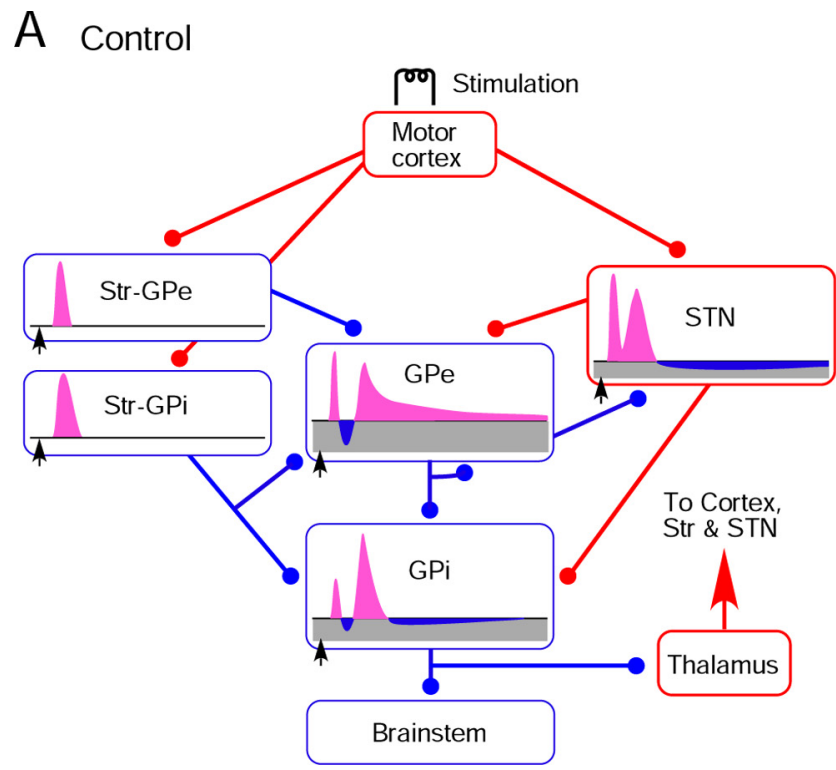

\section{B After DA depletion}

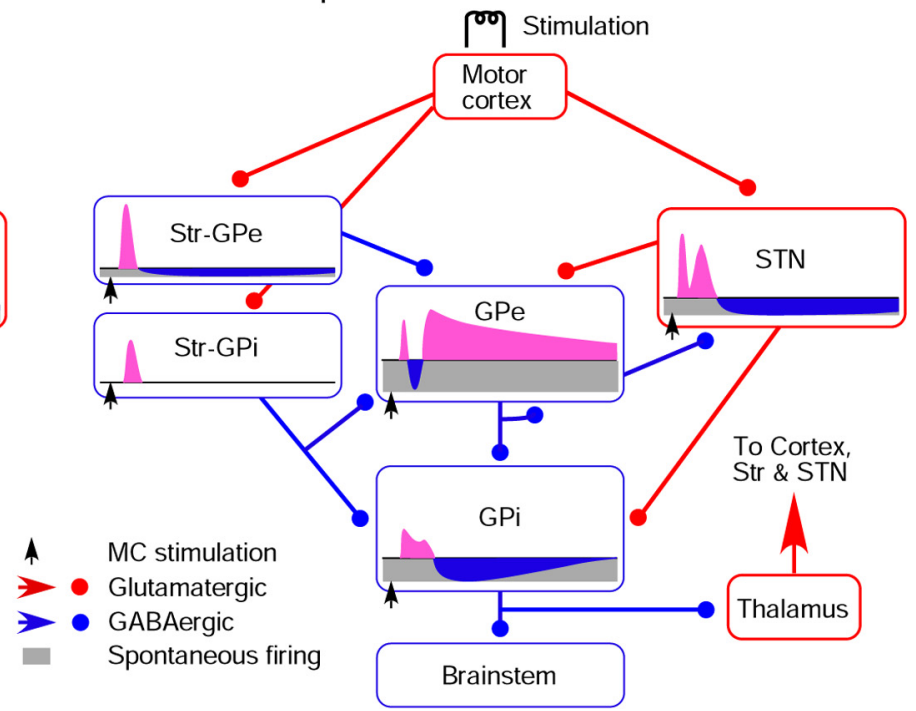

Figure 7. $A, B$, Diagrams show major synaptic connections from MC to GPi and a summary of observations from the present study. The connections are based on the data obtained in previous studies (for review, see Kita, 1994, 2007). MC provides strong inputs to Str and STN. In the control group, MC stimulation induces a shorter latency excitation in STN compared with Str due to the unique membrane properties of STN neurons (Farries et al., 2010). The early excitation in STN evokes an early excitation in GPe and GPi before the Str-mediated inhibition begins. The MC-induced excitation in Str neurons projecting to GPi has a longer duration than that in Str neurons projecting to GPe under normal conditions (Flores-Barrera et al., 2010). The late excitation in STN, which may be formed by various forces, drives the late excitation in GPe and GPi. B, In 6-0HDA rats, MC-induced excitation in Str neurons projecting to GPi decreased (Flores-Barrera et al., 2010). Also, spontaneous firing of Str neurons projecting to GPe increased, which induced a tonic inhibition and disinhibition in GPe neurons. The mean latency of the excitation evoked in those neurons was significantly shorter than in the control group and, as a consequence, the early excitation in GPe was reduced. These changes produce a cascade of changes in GPe and GPi. The short inhibition in GPi was almost abolished after DA depletion, and the mechanisms driving this change are still under investigation.

having collateral innervation to GPe (Kawaguchi et al., 1990; Parent et al., 1995). A study using antidromic identification of Str-SNr neurons (Mallet et al., 2006) and the present juxtacellular labeling results suggest that DA depletion activated only Str-GPe neurons. Possible factors for the increased activity include altered synaptic inputs and membrane properties (Hernandez-Lopez et al., 2000; Flores-Barrera et al., 2010; Surmeier et al., 2010) and changes in thalamo-Str inputs.

Under normal conditions, STN-GPe inputs drive the late excitation in GPe (Ryan and Clark, 1991; Kita, 1992; Nambu et al., 2000; Kita et al., 2004). DA depletion decreased the strength of the late excitation in STN and may decrease the STN-GPe excitation. Thus, after DA depletion, the increased MC-StrGPe disinhibition overrides the decreased excitation and forms a stronger and longer late excitation.

After DA depletion, the increased late excitation in GPe may evoke a strong, long inhibition in STN by removing the presynaptic D2-R-mediated suppression of GABAergic inputs (Shen and Johnson, 2000, 2005; Baufreton and Bevan, 2008; Dejean et al., 2008). The reciprocal connections between GPe and STN form negative-feedback circuits. Thus, a long inhibition in STN should provide a disfacilitation to GPe. The present observations suggest that the force driving the late excitation of GPe is much stronger than the GPe-STN-GPe negative feedback input. GPeSTN projections converge (Baufreton et al., 2009), while STNGPe projections diverge (Parent and Hazrati, 1995). Thus, the GPe-STN-GPe negative feedback may be a divergent system that exerts moderate effects on neurons in a much wider area than the area of original signal generation.

After DA depletion, the increased late excitation in GPe and long inhibition in STN provided strong inhibition and disfacilitation, respectively, and together they generated a powerful long inhibition in GPi, suggesting that a seemingly weak, long inhibi- tion evoked in a subpopulation of Str-GPe neurons can be highly amplified in the basal ganglia. The same mechanism may also be inferred to cause the increased pauses and burst generations in the pallidum and STN of parkinsonian subjects. The cortex, the basal ganglia, and the thalamus form loop connections (Albin et al., 1989; Bergman et al., 1994). It remains to be investigated how the loops transmit the altered responses in GPi and shape the responses of basal ganglia nuclei.

\section{DA depletion altered short latency responses in STN and short inhibition in GPi}

DA depletion also virtually eliminated the MC-induced short inhibition in GPi. This is consistent with the report that DA depletion decreases the responsiveness of Str-GPi neurons to excitatory inputs (Flores-Barrera et al., 2010). However, it is uncertain whether this decreased responsiveness alone can lead to the elimination of the inhibition. DA depletion also decreased the MCinduced early and late excitations in STN, contrary to studies showing an increased responsiveness of STN neurons to glutamate (Shen and Johnson, 2005) and showing that STN neurons in PD patients responded strongly to sensory stimulation (Rodriguez-Oroz et al., 2001). We infer that the increased firing in STN is due to increased excitatory inputs and that the decreased MC-induced excitations was due to the increased potassium conductance that underlies the slow firing adaptation (Barraza et al., 2009) and to the increased spontaneous pauses and bursts, which reduce the reliability of evoking the short latency excitations (our unpublished observation).

\section{Effects of intra-Str methyl L-DOPA injection}

Injection of a behaviorally effective dose of methyl-L-DOPA or muscimol into the DA-depleted Str increased the firing rate, decreased the bursts, and decreased the MC-induced late excitation 
in GPe, indicating that both drugs decreased the background Str-GPe inputs. We infer that these changes in GPe contributed to the decreased bursts and decreased long inhibition in GPi observed after intra-Str L-DOPA. The decreased burst activity is consistent with the suggestion that the same mechanisms that evoke strong, long inhibition in GPi contribute to generation of strong bursts in GPi.

DA depletion increases D2-R expression in Str-GPe neurons (Gerfen et al., 1990) and also sensitizes D2-Rs on cortico-Str terminals (Bamford et al., 2004). Thus, activation of these D2-Rs by DA converted from L-DOPA in Str may underlie the observed effects (Xu and Dluzen, 1996; Tanaka et al., 1999). However, intra-Str methyl-L-DOPA had a minor effect on the restoration of the short inhibition in GPi. The present results suggest that the increased ambulatory movements after acute replacement of DA were due to the reduced late inhibition or reduced burst activity in GPi.

A study in MPTP (1-methyl-4-phenyl-1,2,3,6-tetrahydropyridine)treated late-stage parkinsonian monkeys with fully developed L-DOPA-induced dyskinesias showed that Str activity was significantly higher than normal, and an improvement of movement by systemic L-DOPA injection was associated with an additional increase in Str activity (Liang et al., 2008). We speculate that the L-DOPA-induced increase in Str activity was due to altered expressions of various receptors and ionic channels in the basal ganglia of animals with L-DOPA-induced dyskinesias (Gerfen et al., 2002).

\section{Firing rates and burst index}

In this study, the average firing rate of GPe did not change significantly despite the increased Str-GPe inhibition and a downregulation of $\mathrm{HCN} 2$ channel expressions in GPe that normally enhance autonomous firing (Chan et al., 2011). These increased inhibitory forces were inferred to be counterbalanced by increased excitatory inputs from STN, which may due to increased excitatory inputs, increased rebounds after pauses, and/or a removal of presynaptic D2-R-mediated suppression of synaptic transmissions (Shen and Johnson, 2000, 2005; Baufreton and Bevan, 2008; Dejean et al., 2008). The firing rate of GPi neurons was unchanged despite significantly increased STN activity, probably because of frequent short pauses in firing generated by strongly bursting GPe neurons and an intrinsic adaptation mechanism (Nakanishi et al., 1991). After DA depletion, GPe activity seems to be controlled by increased inhibitory and excitatory forces. Studies in animal models of PD reported no change in the average firing rate of GPe and GPi and an increase in that of STN neurons (Filion, 1979; DeLong, 1990; Hollerman and Grace, 1992; Bergman et al., 1994; Ni et al., 2000; Magill et al., 2001), while others reported a significant decrease in the firing rate of GPe and a significant increase in that of GPi neurons (Albin et al., 1989; DeLong, 1990; Filion and Tremblay, 1991; Mallet et al., 2008; for review, see Wichmann and DeLong, 2006). We infer that the latter observations may be due to a slight dominance of the inhibitory force occurring in GPe at an early stage of DA depletion (Pan and Walters, 1988).

\section{Functional implication}

The most striking finding in the present study was that a seemingly weak, long inhibition evoked in a subpopulation of neurons in DA-depleted Str can be highly amplified in the basal ganglia and generate abnormal responses in GPi, implying that the manner of signal transfer from MC to the basal ganglia output nucleus GPi changed substantially. Under normal conditions, movement- related excitations of MC are transmitted through quiescent Str neurons and induce a short inhibition in GPe. After DA depletion, the MC excitation induces a shorter latency short inhibition followed by a long disinhibition in GPe. This abnormal response sequence in GPe is directly and multisynaptically transmitted to GPi and generates an abnormally strong long inhibition in GPi, which then generates a strong and long excitation in the thalamic projection sites that are transmitted to the MC with incorrect timing and information. It is possible that these changes contribute to the strong synchronization of a wide area of cortex, which leads to abnormal muscle contractions such as rigidity and frozen posture (Goldberg et al., 2002; Pelled et al., 2002).

Another striking finding was that DA depletion virtually eliminated MC-induced short inhibition in GPi and that intra-Str injection of a behaviorally effective dose of methyl-L-DOPA only partially restored the inhibition. Methyl-L-DOPA effectively reduced the abnormal long duration responses and burst generation in the pallidum and STN. Therefore, the generation of abnormal signals in the basal ganglia may underlie the manifestation of some parkinsonian symptoms, and complete restoration of the Str-GPi input may not be required for restoration of some functionality, such as ambulatory movements.

\section{References}

Albin RL, Young AB, Penney JB (1989) The functional anatomy of basal ganglia disorders. Trends Neurosci 12:366-375.

Bamford NS, Zhang H, Schmitz Y, Wu NP, Cepeda C, Levine MS, Schmauss C, Zakharenko SS, Zablow L, Sulzer D (2004) Heterosynaptic dopamine neurotransmission selects sets of corticostriatal terminals. Neuron 42:653-663.

Barraza D, Kita H, Wilson CJ (2009) Slow spike frequency adaptation in neurons of the rat subthalamic nucleus. J Neurophysiol 102:3689-3697.

Baufreton J, Bevan MD (2008) D2-like dopamine receptor-mediated modulation of activity-dependent plasticity at GABAergic synapses in the subthalamic nucleus. J Physiol 586:2121-2142.

Baufreton J, Kirkham E, Atherton JF, Menard A, Magill PJ, Bolam JP, Bevan MD (2009) Sparse but selective and potent synaptic transmission from the globus pallidus to the subthalamic nucleus. J Neurophysiol 102:532-545.

Bergman H, Wichmann T, Karmon B, DeLong MR (1994) The primate subthalamic nucleus. II. Neuronal activity in the MPTP model of parkinsonism. J Neurophysiol 72:507-520.

Bevan MD, Magill PJ, Terman D, Bolam JP, Wilson CJ (2002) Move to the rhythm: oscillations in the subthalamic nucleus-external globus pallidus network. Trends Neurosci 25:525-531.

Bevan MD, Atherton JF, Baufreton J (2006) Cellular principles underlying normal and pathological activity in the subthalamic nucleus. Curr Opin Neurobiol 16:621-628.

Boraud T, Bezard E, Bioulac B, Gross CE (2002) From single extracellular unit recording in experimental and human Parkinsonism to the development of a functional concept of the role played by the basal ganglia in motor control. Prog Neurobiol 66:265-283.

Chan CS, Glajch KE, Gertler TS, Guzman JN, Mercer JN, Lewis AS, Goldberg $\mathrm{AB}$, Tkatch T, Shigemoto R, Fleming SM, Chetkovich DM, Osten P, Kita H, Surmeier DJ (2011) HCN channelopathy in external globus pallidus neurons in models of Parkinson's disease. Nat Neurosci 14:85-92.

Cooper AJ, Stanford IM (2001) Dopamine D2 receptor mediated presynaptic inhibition of striatopallidal GABA(A) IPSCs in vitro. Neuropharmacology 41:62-71.

Dejean C, Gross CE, Bioulac B, Boraud T (2008) Dynamic changes in the cortex-basal ganglia network after dopamine depletion in the rat. J Neurophysiol 100:385-396.

DeLong MR (1971) Activity of pallidal neurons during movement. J Neurophysiol 34:414-427.

DeLong MR (1990) Primate models of movement disorders of basal ganglia origin. Trends Neurosci 13:281-285.

Farries MA, Kita H, Wilson CJ (2010) Dynamic spike threshold and zero membrane slope conductance shape the response of subthalamic neurons to cortical input. J Neurosci 30:13180-13191. 
Filion M (1979) Effects of interruption of the nigrostriatal pathway and of dopaminergic agents on the spontaneous activity of globus pallidus neurons in the awake monkey. Brain Res 178:425-441.

Filion M, Tremblay L (1991) Abnormal spontaneous activity of globus pallidus neurons in monkeys with MPTP-induced parkinsonism. Brain Res 547:142-151.

Floran B, Floran L, Sierra A, Aceves J (1997) D2 receptor-mediated inhibition of GABA release by endogenous dopamine in the rat globus pallidus. Neurosci Lett 237:1-4.

Flores-Barrera E, Vizcarra-Chacón BJ, Tapia D, Bargas J, Galarraga E (2010) Different corticostriatal integration in spiny projection neurons from direct and indirect pathways. Front Syst Neurosci 4:15.

Flores-Hernández J, Galarraga E, Bargas J (1997) Dopamine selects glutamatergic inputs to neostriatal neurons. Synapse 25:185-195.

Fujimoto K, Kita H (1993) Response characteristics of subthalamic neurons to the stimulation of the sensorimotor cortex in the rat. Brain Res 609:185-192.

Gajendiran M, Cepeda C, Kha HT, Sison JD, Levine MS (2005) Electrophysiological alterations in subthalamic neurons after unilateral dopamine depletion in the rat. J Neurosci Res 80:203-210.

Gatev P, Wichmann T (2009) Interactions between cortical rhythms and spiking activity of single basal ganglia neurons in the normal and parkinsonian state. Cereb Cortex 19:1330-1344.

Gerfen CR, Engber TM, Mahan LC, Susel Z, Chase TN, Monsma FJ Jr, Sibley DR (1990) D1 and D2 dopamine receptor-regulated gene expression of striatonigral and striatopallidal neurons. Science 250:1429-1432.

Gerfen CR, Miyachi S, Paletzki R, Brown P (2002) D1 dopamine receptor supersensitivity in the dopamine-depleted striatum results from a switch in the regulation of ERK1/2/MAP kinase. J Neurosci 22:5042-5054.

Goldberg JA, Boraud T, Maraton S, Haber SN, Vaadia E, Bergman H (2002) Enhanced synchrony among primary motor cortex neurons in the 1-methyl-4-phenyl-1,2,3,6-tetrahydropyridine primate model of Parkinson's disease. J Neurosci 22:4639-4653.

Hernandez-Lopez S, Tkatch T, Perez-Garci E, Galarraga E, Bargas J, Hamm H, Surmeier DJ (2000) D2 dopamine receptors in striatal medium spiny neurons reduce L-type $\mathrm{Ca} 2+$ currents and excitability via a novel PLC[beta] 1-IP3-calcineurin-signaling cascade. J Neurosci 20:8987-8995.

Hollerman JR, Grace AA (1992) Subthalamic nucleus cell firing in the 6-OHDA-treated rat: basal activity and response to haloperidol. Brain Res 590:291-299.

Israel Z, Bergman H (2008) Pathophysiology of the basal ganglia and movement disorders: from animal models to human clinical applications. Neurosci Biobehav Rev 32:367-377.

Kawaguchi Y, Wilson CJ, Emson PC (1990) Projection subtypes of rat neostriatal matrix cells revealed by intracellular injection of biocytin. J Neurosci 10:3421-3438.

Kelland MD, Soltis RP, Anderson LA, Bergstrom DA, Walters JR (1995) In vivo characterization of two cell types in the rat globus pallidus which have opposite responses to dopamine receptor stimulation: comparison of electrophysiological properties and responses to apomorphine, dizocilpine, and ketamine anesthesia. Synapse 20:338-350.

Kita H (1992) Responses of globus pallidus neurons to cortical stimulation: intracellular study in the rat. Brain Res 589:84-90.

Kita H (1994) Physiology of two disynaptic pathways from the sensorimotor cortex to the basal ganglia output nuclei. In: The basal ganglia IV (Percheron G, McKenzie JS, Feger J, eds), pp 263-276. New York: Plenum

Kita H (2007) Globus pallidus external segment. Prog Brain Res 160:111-133.

Kita H, Nambu A, Kaneda K, Tachibana Y, Takada M (2004) Role of ionotropic glutamatergic and GABAergic inputs on the firing activity of neurons in the external pallidum in awake monkeys. J Neurophysiol 92:3069-3084.

Kitai ST, Deniau JM (1981) Cortical inputs to the subthalamus: intracellular analysis. Brain Res 214:411-415.

Legéndy CR, Salcman M (1985) Bursts and recurrences of bursts in the spike trains of spontaneously active striate cortex neurons. J Neurophysiol 53:926-939.

Liang L, DeLong MR, Papa SM (2008) Inversion of dopamine responses in striatal medium spiny neurons and involuntary movements. J Neurosci 28:7537-7547.

Magill PJ, Bolam JP, Bevan MD (2001) Dopamine regulates the impact of the cerebral cortex on the subthalamic nucleus-globus pallidus network. Neuroscience 106:313-330.

Mallet N, Ballion B, Le Moine C, Gonon F (2006) Cortical inputs and GABA interneurons imbalance projection neurons in the striatum of parkinsonian rats. J Neurosci 26:3875-3884.

Mallet N, Pogosyan A, Márton LF, Bolam JP, Brown P, Magill PJ (2008) Parkinsonian beta oscillations in the external globus pallidus and their relationship with subthalamic nucleus activity. J Neurosci 28:1424514258.

Maurice N, Deniau JM, Menetrey A, Glowinski J, Thierry AM (1998) Prefrontal cortex-basal ganglia circuits in the rat: involvement of ventral pallidum and subthalamic nucleus. Synapse 29:363-370.

Nakanishi H, Kita H, Kitai ST (1991) Intracellular study of rat entopeduncular nucleus neurons in an in vitro slice preparation: response to subthalamic stimulation. Brain Res 549:285-291.

Nambu A, Tokuno H, Hamada I, Kita H, Imanishi M, Akazawa T, Ikeuchi Y, Hasegawa N (2000) Excitatory cortical inputs to pallidal neurons via the subthalamic nucleus in the monkey. J Neurophysiol 84:289-300.

Nambu A, Tachibana Y, Kaneda K, Tokuno H, Takada M (2005) Dynamic model of basal ganglia functions and parkinson's disease. In: The basal ganglia VIII (Bolam JP, Ingham CA, Magill PJ, eds), pp 307-312. New York: Springer

Ni Z, Bouali-Benazzouz R, Gao D, Benabid AL, Benazzouz A (2000) Changes in the firing pattern of globus pallidus neurons after the degeneration of nigrostriatal pathway are mediated by the subthalamic nucleus in the rat. Eur J Neurosci 12:4338-4344.

Pan HS, Walters JR (1988) Unilateral lesion of the nigrostriatal pathway decreases the firing rate and alters the firing pattern of globus pallidus neurons in the rat. Synapse 2:650-656.

Pang Z, Ling GY, Gajendiran M, Xu ZC (2001) Enhanced excitatory synaptic transmission in spiny neurons of rat striatum after unilateral dopamine denervation. Neurosci Lett 308:201-205.

Parent A, Hazrati LN (1995) Functional anatomy of the basal ganglia. II. The place of subthalamic nucleus and external pallidum in basal ganglia circuitry. Brain Res Brain Res Rev 20:128-154.

Parent A, Charara A, Pinault D (1995) Single striatofugal axons arborizing in both pallidal segments and in the substantia nigra in primates. Brain Res 698:280-284.

Pelled G, Bergman H, Goelman G (2002) Bilateral overactivation of the sensorimotor cortex in the unilateral rodent model of Parkinson's disease: a functional magnetic resonance imaging study. Eur J Neurosci 15:389-394.

Pinault D (1996) A novel single-cell staining procedure performed in vivo under electrophysiological control: morpho-functional features of juxtacellularly labeled thalamic cells and other central neurons with biocytin or Neurobiotin. J Neurosci Methods 65:113-136.

Rodriguez-Oroz MC, Rodriguez M, Guridi J, Mewes K, Chockkman V, Vitek J, DeLong MR, Obeso JA (2001) The subthalamic nucleus in Parkinson's disease: somatotopic organization and physiological characteristics. Brain 124:1777-1790.

Ryan LJ, Clark KB (1991) The role of the subthalamic nucleus in the response of globus pallidus neurons to stimulation of the prelimbic and agranular frontal cortices in rats. Exp Brain Res 86:641-651.

Ryan LJ, Clark KB (1992) Alteration of neuronal responses in the subthalamic nucleus following globus pallidus and neostriatal lesions in rats. Brain Res Bull 29:319-327.

Shen KZ, Johnson SW (2000) Presynaptic dopamine D2 and muscarine M3 receptors inhibit excitatory and inhibitory transmission to rat subthalamic neurones in vitro. J Physiol 525:331-341.

Shen KZ, Johnson SW (2005) Dopamine depletion alters responses to glutamate and GABA in the rat subthalamic nucleus. Neuroreport 16:171-174.

Shen W, Flajolet M, Greengard P, Surmeier DJ (2008) Dichotomous dopaminergic control of striatal synaptic plasticity. Science 321:848-851.

Shin RM, Masuda M, Miura M, Sano H, Shirasawa T, Song WJ, Kobayashi K, Aosaki T (2003) Dopamine D4 receptor-induced postsynaptic inhibition of GABAergic currents in mouse globus pallidus neurons. J Neurosci 23:11662-11672.

Spencer JP, Murphy KP (2000) Bi-directional changes in synaptic plasticity induced at corticostriatal synapses in vitro. Exp Brain Res 135:497-503.

Surmeier DJ, Shen W, Day M, Gertler T, Chan S, Tian X, Plotkin JL (2010) The role of dopamine in modulating the structure and function of striatal circuits. Prog Brain Res 183:149-167.

Tanaka H, Kannari K, Maeda T, Tomiyama M, Suda T, Matsunaga M (1999) 
Role of serotonergic neurons in L-DOPA-derived extracellular dopamine in the striatum of 6-OHDA-lesioned rats. Neuroreport 10:631-634.

Tseng KY, Kasanetz F, Kargieman L, Riquelme LA, Murer MG (2001) Cortical slow oscillatory activity is reflected in the membrane potential and spike trains of striatal neurons in rats with chronic nigrostriatal lesions. J Neurosci 21:6430-6439.

Watanabe K, Kita T, Kita H (2009) Presynaptic actions of d2-like receptors in the rat cortico-striato-globus pallidus disynaptic connection in vitro. J Neurophysiol 101:665-671.

West AR, Grace AA (2002) Opposite influences of endogenous dopamine D1 and D2 receptor activation on activity states and electrophysiological properties of striatal neurons: studies combining in vivo intracellular recordings and reverse microdialysis. J Neurosci 22:294-304.

Wichmann T, DeLong MR (2006) Basal ganglia abnormalities in Parkinson's disease. J Neural Transm Suppl 70:21-25.

Wichmann T, Soares J (2006) Neuronal firing before and after bursts in the monkey basal ganglia is predictably patterned in the normal state and altered in parkinsonism. J Neurophysiol 95:2120-2133.

Wichmann T, Bergman H, DeLong MR (1994) The primate subthalamic nucleus. I. Functional properties in intact animals. J Neurophysiol 72: 494-506.

Wilson CJ, Chang HT, Kitai ST (1983) Disfacilitation and long-lasting inhibition of neostriatal neurons in the rat. Exp Brain Res 51:227-235.

Xu K, Dluzen DE (1996) L-DOPA modulation of corpus striatal dopamine and dihydroxyphenylacetic acid output from intact and 6-OHDA lesioned rats. J Neural Transm 103:1295-1305.

Yoon KW, Canney DJ, Covey DF, Rothman SM (1990) Modulation of the picrotoxin receptor by fluorinated ethyl, methyl-butyrolactones. J Pharmacol Exp Ther 255:248-255.

Yoshida S, Nambu A, Jinnai K (1993) The distribution of the globus pallidus neurons with input from various cortical areas in the monkeys. Brain Res 611:170-174. 